\title{
NEUROIMAGING IN NEONATAL ENCEPHALOPATHY
}

Investigators at St Francis Medical Center, Cape Girardeau, MO, and multiple centers in the US, using a database from the Vermont Oxford Network Registry, studied the pattern of use and findings of computed tomography, MRI, and intracranial ultrasound in the evaluation of infants with neonatal encephalopathy. CT scans were performed on 933 of 4107 (22.7\%) infants, and 100 of 921 (10.9\%) of those received multiple CT scans. Compared with MRI, CT provided less detailed evaluation of cerebral injury, but was more sensitive than cranial ultrasound for hemorrhage and deep brain structural abnormalities. Despite concerns over potential harm from radiation exposure and a diagnostic value inferior to that of MRI, CT is commonly used for neonatal imaging. Data collected in this study suggest that cranial ultrasound for screening, followed by MRI is more appropriate than CT at any stage of evaluation of infants with neonatal encephalopathy. (Barnette AR, Horbar JD, Soll RF, et al. Neuroimaging in the evaluation of neonatal encephalopathy. Pediatrics 2014 May 26;133(6):e1508-17).

COMMENTARY. Fetal exposure to radiation is linked to neurologic abnormalities [1], and infant medical radiation exposure is associated with impaired cognitive development [2]. The Image Gently education campaign in radiology promotes reduction of the frequency of CT scanning [3], but further efforts by caregivers of newborns are needed to limit the use of CT in evaluation of neonatal encephalopathy [4].

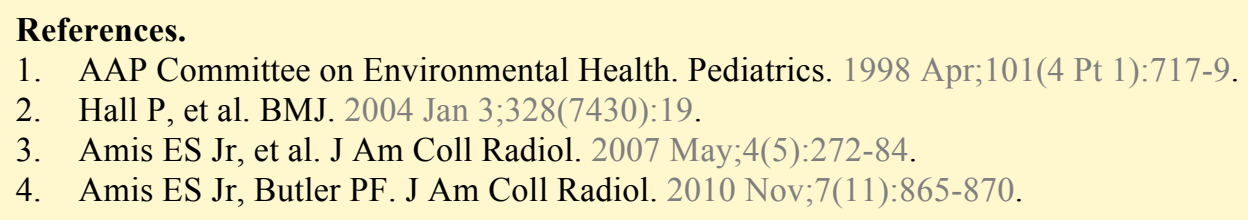

\section{AUTOIMMUNE DISORDERS}

\section{CELIAC DISEASE AND OCCIPITAL EPILEPSY}

Investigators at University of Gaziantep, Turkey, studied the frequency of celiac disease (CD) among children with occipital epilepsy. Two out of 90 epilepsy patients with occipital discharges were tissue transglutaminase (tTG) positive, a prevalence of $1 / 45(2.22 \%)$, and diagnosis of $\mathrm{CD}$ was confirmed by endoscopic duodenal biopsy. In patients with occipital lobe epilepsy associated with $\mathrm{CD}$, seizures were uncontrolled with monotherapy (oxcarbamazepine or valproic acid). Screening for $\mathrm{CD}$ is recommended in children with resistant occipital epilepsy. (Dai AI, Akcali A, Varan C, Demiryurek AT. Prevalence of resistant occipital lobe epilepsy associated with celiac disease in children. Childs Nerv Syst 2014 Jun;30(6):1091-8).

COMMENTARY. Neurological diseases associated with $\mathrm{CD}$ in addition to occipital epilepsy include cerebellar ataxia, peripheral neuropathy, myositis, neuromyotonia, myasthenic syndrome, cerebral calcification, myelopathy, and dementia. Reports of the prevalence of CD in children with occipital epilepsy are variable and 